\title{
POTENTIAL OF TWIN-SCREW COMPRESSOR AS VIBRATION SOURCE FOR ENERGY HARVESTING APPLICATIONS
}

\author{
Claudia BORZEA ${ }^{1,2}$, Valentin PETRESCU ${ }^{1,2}$, Iulian VLĂDUCĂ ${ }^{1}$, Mihaela $\operatorname{ROMAN}^{1,2}$, \\ Gabriel BADEA ${ }^{1,2}$ \\ ${ }^{1}$ Romanian Research and Development Institute for Gas Turbines COMOTI \\ ${ }^{2}$ University Politehnica of Bucharest \\ claudia.borzea@,comoti.ro
}

\begin{abstract}
The paper presents the vibrational behaviour of a twin-screw compressor at various functioning regimes. The speed is varied by means of a $250 \mathrm{~kW}$ electric driving motor, via a frequency converter. The compressor can supply pressurized air up to $\sim 16$ bar into a buffer tank. The air is used for testing various equipment on test bench, such as expanders, pneumatic starters for gas turbine engines, or other compressors and blowers used for compressing air starting from a pressure higher than the atmospheric pressure. Like all other industrial equipment, compressors experience inherent vibrations in operation. The pulsatory effects of the air within the compressor also give birth to harmonics. The study herein involves gathering vibration data and represent it as vibration spectra. Raw vibration signals, employed for assessing the condition of the machinery, may not always be clear in the frequency spectrum for vibration analysis, thus being required to apply Fast Fourier Transform for converting the time waveform into frequency spectrum. The study herein pursues a future application of energy harvesting from vibrations, and outlines that the spectral analysis of the compressor is convenient for piezoelectric cantilever harvesters in terms of vibration frequency and amplitude.
\end{abstract}

\section{INTRODUCTION}

Energy harvesting from vibrations has attracted an increasing research interest is the past several years, seeking methods of accumulating and storing energy from residual sources for powering electronic devices. Micro-energy harvesting technologies that rely on typical mechanical and thermal sources is quite low, the harvested power range varying from the order of microwatts to milliwatts.

When occurring in machinery such as compressors, vibrations indicate potential inchoate faults or emerging harms of rotating mechanical parts (rotors, bearings etc.) particularly [1-5]. Vibration can usually be caused by unbalance, shafts misalignment, screws looseness, bearing issues or resonances at critical speeds.

Harnessing energy from industrial machinery offers the challenge for conducting research in the field, exploiting inherent vibration and heating effects [6], since this type of energy generation technology is actively developing at the present moment and has a long way until reaching a satisfying technological maturity [7].

Equipment vibration can also cause noise when reaching audible frequencies and can affect not only the machine's service life, but can also cause irreversible damage to operating personnel's hearing. During compressors start-up, operation and shutdown, it is a must to wear noise protection ear muffs or plugs.

Piezoelectric devices have a high energy conversion efficiency, however this only at resonance. Outside resonant frequency condition, for a cantilever beam with an inertial mass placed on its free end, the electric response is very poor [6].

The aim for harvesting vibration energy is mainly due to eliminating the need for cabling if the site has difficult conditions for adding extra cabling. A very relevant example is the retrofitting of the propulsion system of a ship [8]. A wireless transmission from sensors would 
be extremely beneficial as it would spare a lot of effort and time, as well as the extra costs for special cables, strict navy arrangements and requirements, as well as man work. Another application would be powering sensor nodes in hazardous locations, subjected to regulations for using costly ATEX certified equipment and cabling in the field [9].

\section{TWIN-SCREW COMPRESSOR SKID}

A twin-screw compressor unit (Fig. 1a) is a rotary bladed machine, categorised as positive displacement compressor, which operates by means of two rotors (male and female) that reduce the volume of the gas trapped between them progressively as gas moves along the intermeshed rotors (Fig. 1b). The volume reduction results in the gas compression. Air compressors can be used to drive pneumatic starters, compressors and blowers that elevate pressure from a value above atmospheric pressure, for providing industrial or instrumental air for pneumatic tools, and so on, in various applications [10].

a)

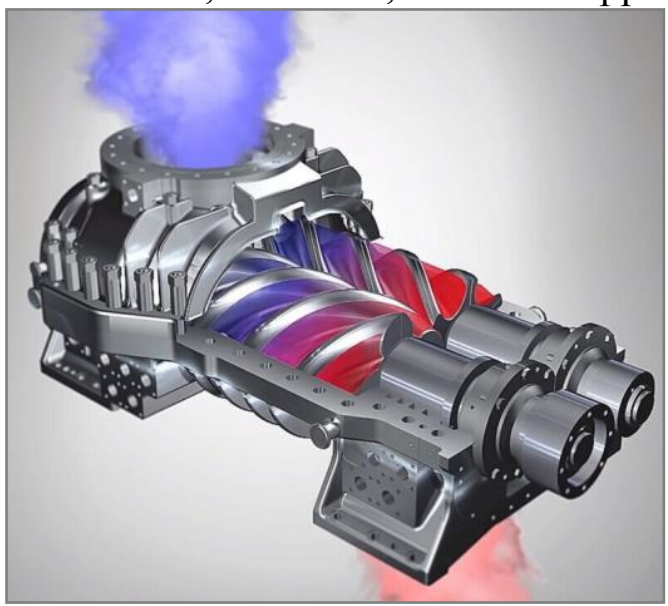

b)

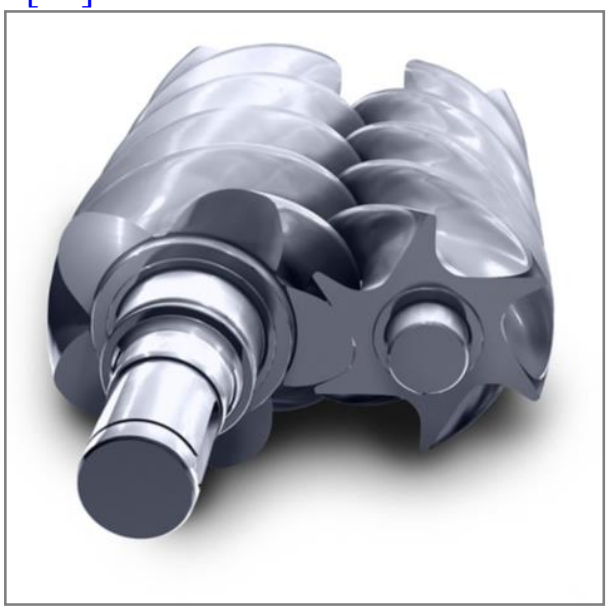

Fig. 1. Twin-screw compressor: a) principle of operation [11]; b) intermeshing rotors profiles [12]

The purpose of subjecting the compressor to measurements on a test bed (Fig. 2) is to detect inchoate faults such as shafts misalignments, poor screws tightening, potential gripping of the rotors, and test the lubrication installation to avoid friction between the rotors.

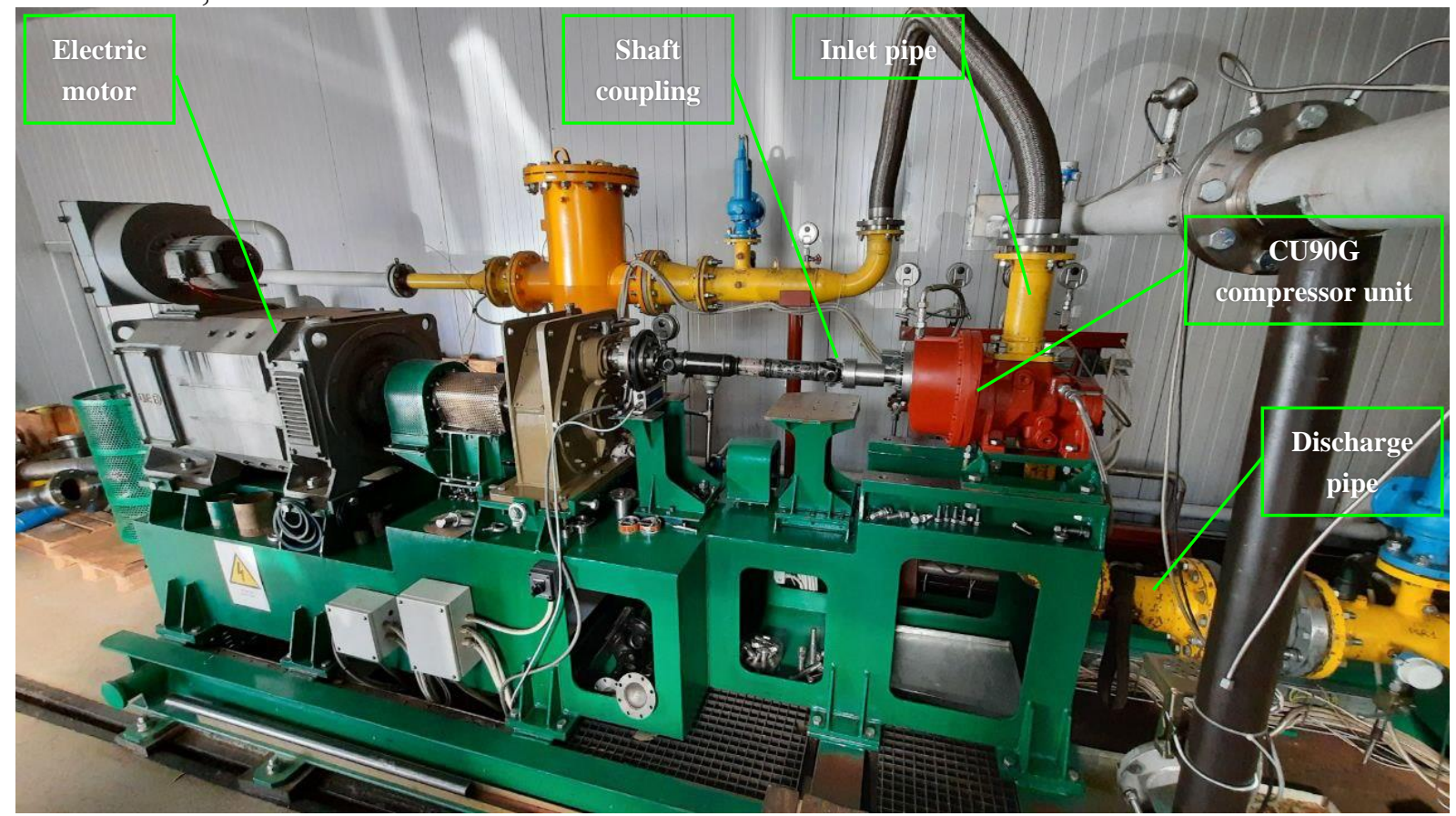

Fig. 2. CU series compressor test bed 
The CU90G compressor performances are presented in Fig. 3 below, indicating the maximum rotational speeds of the male rotor for all driver rotational speeds ( $1450 \mathrm{rpm}$ to 3550 $\mathrm{rpm}$ ), for achieving the discharge pressure desired, as declared in compressor manual [13].

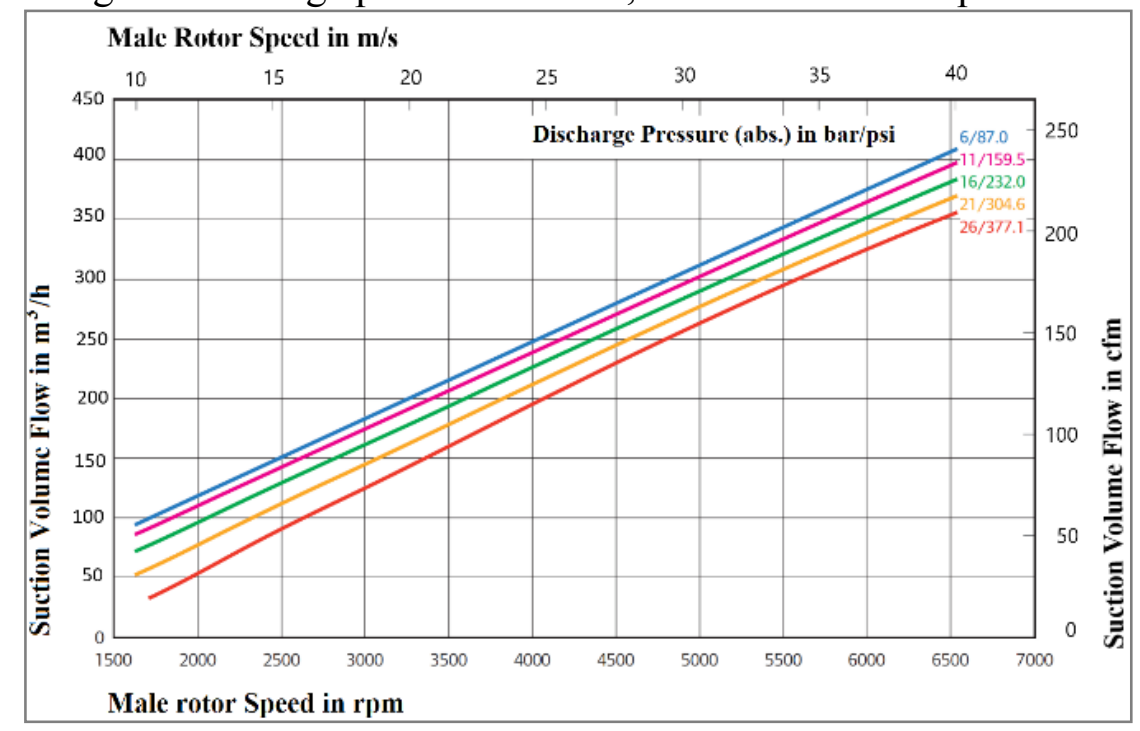

Fig. 3. CU90G compressor performance curves [13]

\section{TWIN-SCREW COMPRESSOR VIBRATIONS}

According to the standard ISO 10816-3, machines are classified differently depending on their mounting, if it is considered to be flexible or rigid. This aspect affects to great extent the machines' vibration and resonant frequencies, which are related to the basic running speeds of the compressor in the paper herein. For instance, the resonances of a so-called flexible machine, which is provided with an elastic layer or springs underneath, occur at lower running speeds, starting to vibrate at lower rotational speeds. When increasing the speed and the machine is not vibrating at a frequency matching one of its resonances, the vibration level (magnitude) is reduced. Modern machines that have high speeds and flexible bearing supports and foundations, are considered flexible [14].

Even in this case, due to many parts and connected shafts, multiple resonances exist in the case of a compressor skid. Vibrations occur both due to the compressor itself, as well as because of slight misalignments at shafts coupling, bearings and other joints. The highest vibrations for the compressor presented are generated by the rotational speed of its male rotor. The vibration frequency depends on the number of lobes and when designing the compressor installation, it is a must to consider avoiding the resonant frequencies with the rotational speeds of male and female rotors that can jeopardise the safe operation.

Screw compressors produce strong pulsations during operation, leading to strong vibrations and noise in the upstream and downstream piping system. International standards recommend, in this case, a study of the angular (rotational) frequencies in terms of amplitude and main components of the frequency spectrum, including the first harmonics. The identification of the amplitudes of the fundamental frequency and of the harmonics is realised by means of applying FFT (Fast Fourier Transform) analysis.

By varying the operating speed of the compressor, the frequency spectrum components can be visualised, and the resonances of the compressor installation can be determined. The maximum amplitude is generated by a relative rotational resonance between male and female rotors, and it can be determined by using the phase difference detecting method between two sinusoidal tooth passing signals from involute gears attached to each rotor. The pulsation magnitude increases with the operating speeds for the discharge pressure pulsations. Pressure 
bursts and pressure differences of trapped volumes of gas also give birth to shocks on longitudinal axis along shaft axis. This transient pulsatory behaviour due to air compression process determine higher harmonics, multiplying the fundamental frequency.

\section{VIBRATION MEASUREMENT AND ACQUIRED DATA ANALYSIS}

The vibration measurements were conducted using a vibration analyser for industrial purposes (Fig. 4). VIBER X5 MK III analyser is an advanced vibration tool, which provides a complete system for the predictive maintenance of machinery, together with its software. This one is capable of measuring, processing, displaying and storing a wide range of measurement parameters. Various types of field data can be acquired, such as vibration information, bearing analysis, temperature, speed and other process variables that can expand its analysis capabilities for a specific process. The device can also be used for one or two plane balancing [14].

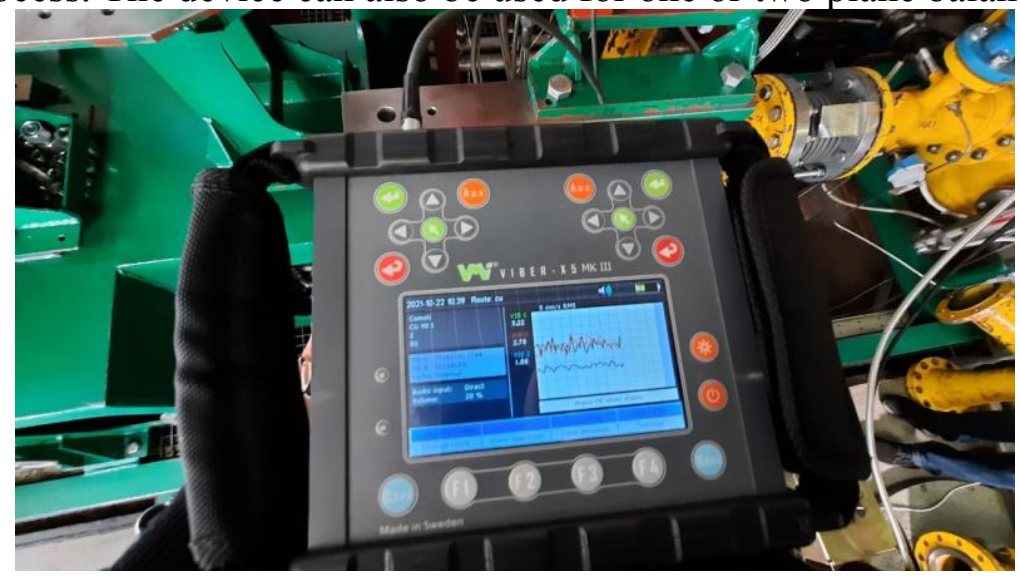

Fig. 4. Viber X5 MK III vibration analyser

For measuring compressor vibrations, a triaxial accelerometer was used and connected via cable to the vibration analyser. In the paper herein, only Point 1 where it would be easiest to place piezoelectric harvesters has been discussed (Fig. 5). The compressor was driven at a variable speed via a frequency converter varying the speed of the DC driving motor. The paper exemplifies the compressor driven at $2500 \mathrm{rpm}$, controllably reducing the section of the motor operated discharge valve, so as to create a pressure of 6 bar and 9 bar respectively. The $\mathrm{Y}$ axis of the accelerometer is considered along the axis of the compressor shaft.

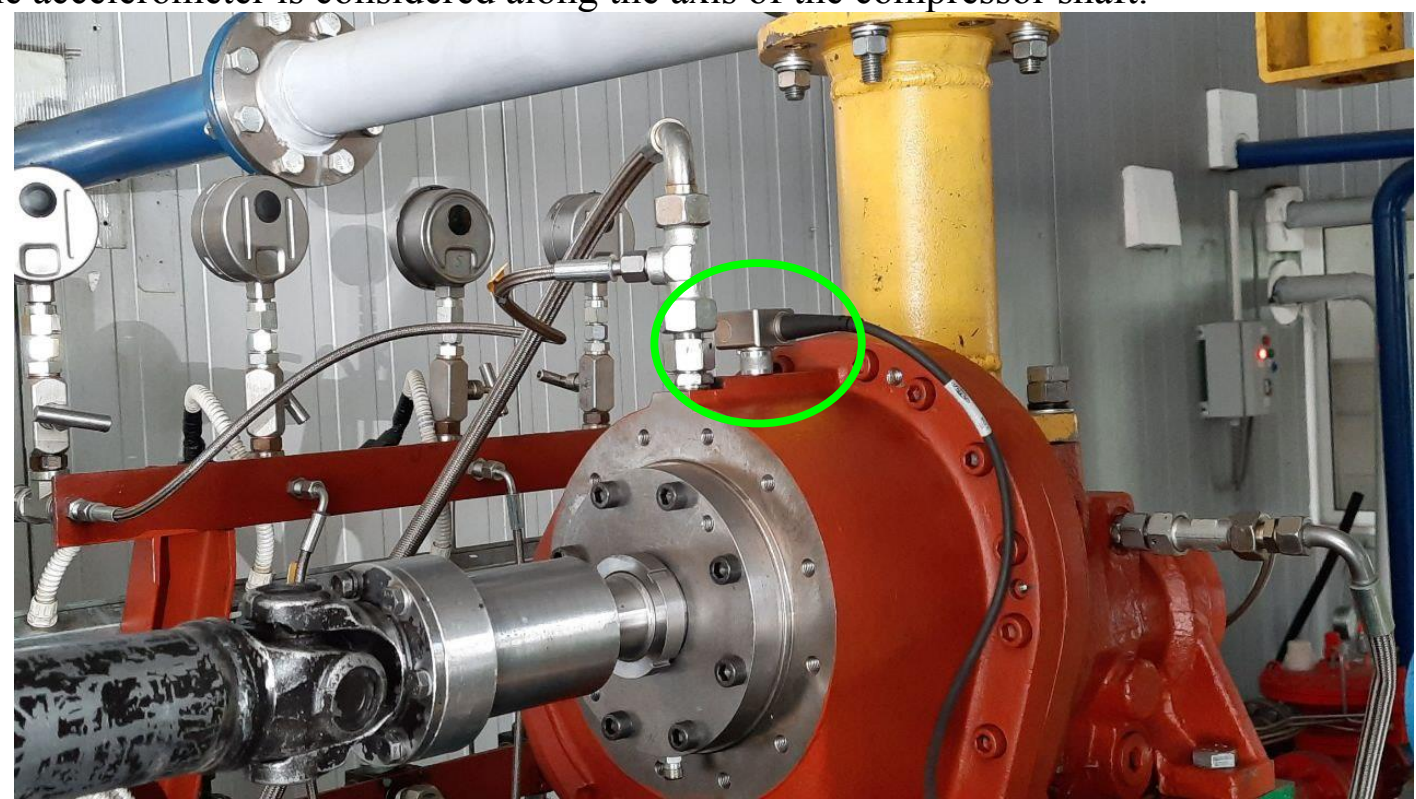

Fig. 5. Triaxial accelerometer placed on compressor housing in measurement spot 1 
The measured vibration data was stored in the memory of the analyser, subsequently saving the data for post-processing and analysis, and for obtaining graphs in a spreadsheet. The frequency spectra after employing the FFT function of the analyser are represented in the following two plots.

Fig. 6 shows the vibration speed function of frequency, for discharge pressure of 6 bar at $2500 \mathrm{rpm}$. The different frequency components are due to the several parts in motion, such as male and female rotors. The fundamental frequency of the compressor is at $423.5 \mathrm{~Hz}$.

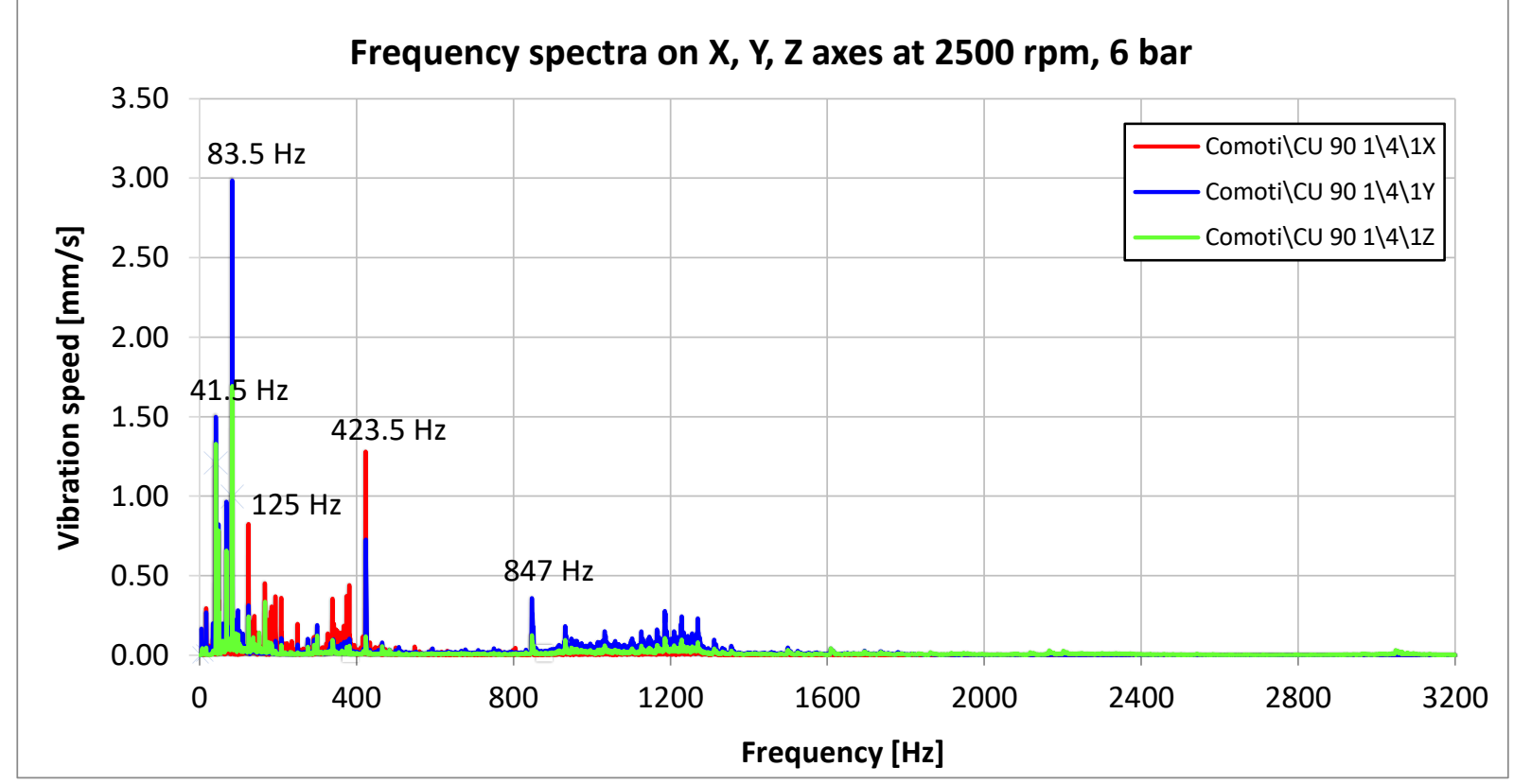

Fig. 6. Vibration spectra in measuring point 1 at 6 bar

Fig. 7 shows the frequency spectra on $\mathrm{X}, \mathrm{Y}$ and $\mathrm{Z}$ directions. The frequencies of the spectral components slightly increase when increasing the pressure to $9 \mathrm{bar}$, while the speed is maintained constant, at the same value of $2500 \mathrm{rpm}$. The fundamental frequency is now found at $425 \mathrm{~Hz}$. The data sampling was conducted with a step of $0.5 \mathrm{~Hz}$ in both cases. It is worthwhile to note that the male and female rotors frequencies remain stable at $83.5 \mathrm{~Hz}$ and $41.5 \mathrm{~Hz}$ respectively, depending only on speed and not also on discharge pressure.

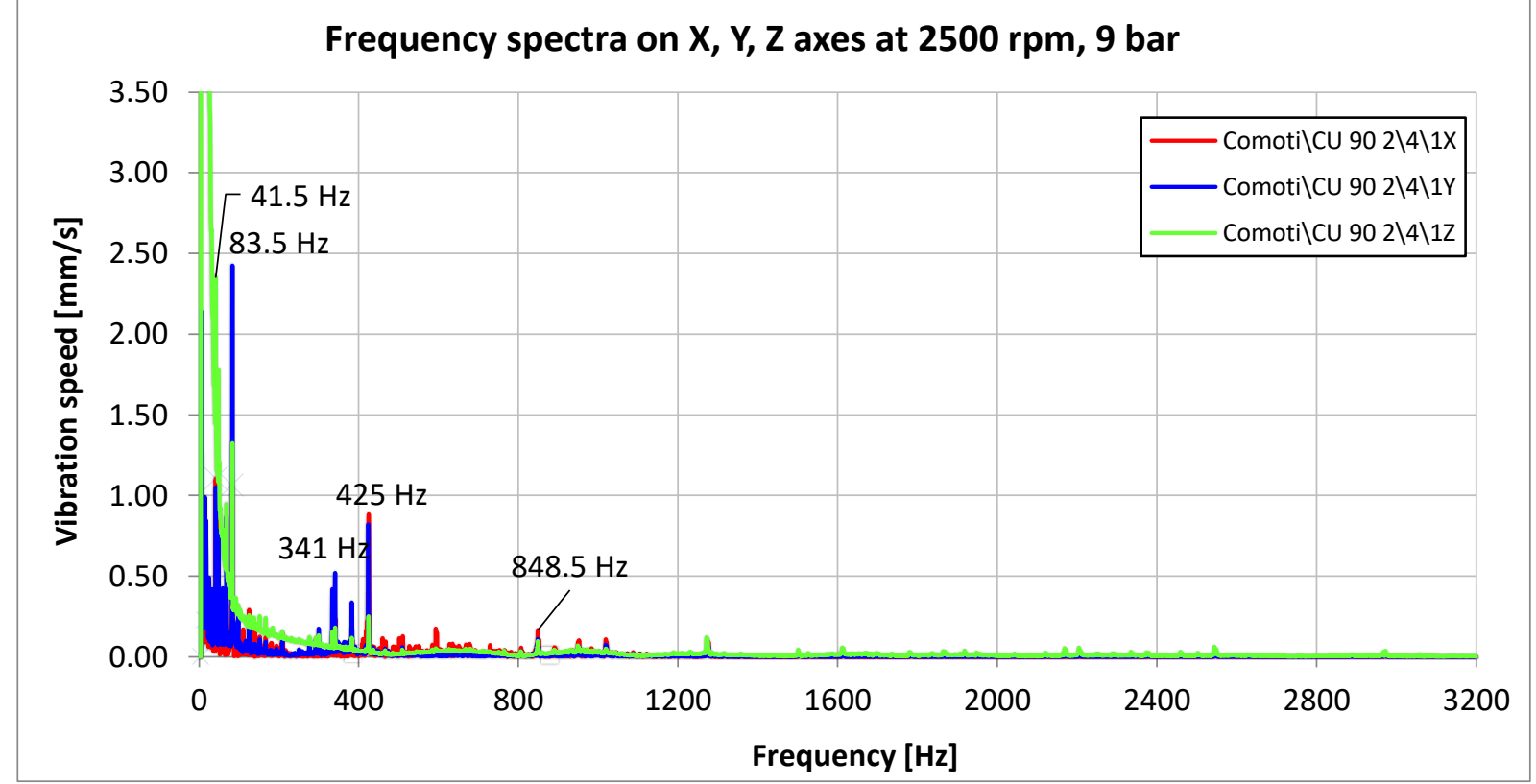

Fig. 7. Vibration spectra in measuring point 1 at 9 bar 


\section{CONCLUSIONS}

The vibration measurements when testing a compressor are meant to detect incipient faults and problems that can arise when the pulsations give birth to acoustic resonances within the pipes or resonate with the natural frequencies of mechanical structures. The working fluid pulsations at high frequencies inside pipes or vessels, combined with these ones' responses, can also cause harmonics as well as noise problems. Simulation software is used for the numerical computation of acoustic natural frequencies (transversal waves) as well as of mechanical natural frequencies (casing) associated with the oil separator vessel and other components of the skid. Thus, resonance frequencies can be avoided. On the other hand, for harvesting maximum energy, piezoelectric resonant structures ought to operate near resonance. Future research and works shall consider adjusting the natural frequency of physical piezoelectric cantilevers to match male rotor's vibration frequency $(\sim 83.5 \mathrm{~Hz})$, pursued due to both its stability and highest amplitude. For that, vibration spectra will be extracted for other operating modes in terms of speed and pressure.

\section{REFERENCES}

[1] D. Hübel and P. Žitek, "Screw compressor analysis from a vibration point-of-view", 36th Meeting of Departments of Fluid Mechanics and Thermodynamics AIP Conference Proceedings, vol. 1889, p. 020011, 2017. DOI: $10.1063 / 1.5004345$.

[2] Y. Zhao, J. Feng, B. Zhao, S. Zhou, Z. Tang and X. Peng, "Vibration analysis and control of a screw compressor outlet piping system", Proceedings of the Institution of Mechanical Engineers, Part E: Journal of Process Mechanical Engineering, vol. 233, no. 2, pp. 403-411, 2018. 10.1177/0954408918763561.

[3] M. Cerpinska, M. Irbe and R. Elmanis-Helmanis, "Vibration of foundation for rotary screw compressors installed on skid mounting", 17th International Scientific Conference Engineering for Rural Development, pp. 1997-2002, 2018. DOI: 10.22616/ERDEV2018.17.N094.

[4] R. Vaughan, W. Bratek and M. Hahn, "Baseline Testing Provides Opportunities for Continuous Improvement", in Gas Machinery Research Council meeting, Nashville, Tennessee, 2014. https://www.betamachinery.com/assets/pdfs/Technical_Articles/Baseline_Testing_Provides_Opportunities for_Continuous Improvement.pdf.

[5] D. Hübel and P. Žitek, "Screw compressor analysis from a vibration point-of-view", AIP Conference Proceedings, vol. 1889, Issue 1, p. 020011, 2017. DOI: $10.1063 / 1.5004345$.

[6] A. Lenhard, "Review of energy harvesting methods for twin screw extruders", Bachelor of Science Thesis, Massachusetts Institute of Technology, Department of Mechanical Engineering, 2020. https://hdl.handle.net/1721.1/127922.

[7] C. Yang, N. Bin Noor Hanafi, N. Bt Mohamad Hanif, A. Ismail and H. Chang, "A Novel Non-Intrusive Vibration Energy Harvesting Method for Air Conditioning Compressor Unit", Sustainability, vol. 13, no. 18, p. 10300, 2021. DOI: 10.3390/su131810300.

[8] I. Vlăducă, C. Borzea, M. Vasile, G. Bălan, A. Săvescu, A. Mitru, D. Niculescu, C. Nechifor, D. Ușeriu, T. Stănescu, P. Badea and R. Ciobanu, "Automation Control System for Naval Propulsion Retrofitting", 2021 International Conference on Applied and Theoretical Electricity (ICATE), 2021. DOI: 10.1109/ICATE49685.2021.9465065.

[9] C. Nechifor, C. Borzea, A. Stoicescu, D. Lale and M. Vasile, "Modular automation cabinet for proactive monitoring in ATEX Zone 2", in 10th Edition of the International Symposium on Occupational Health and Safety - SESAM 2021, INCD INSEMEX, 2021.

[10]VMAC, "Easy Guide to Rotary Screw Air Compressors (2021 Edition)", vmacair.com, 2021. https://www.vmacair.com/blog/easy-guide-to-rotary-screw-air-compressors-for-trucks-vans/.

[11] Y. Kheng, "Why is there water in my air compressor? - Mc-Cast Engineering", Mc-Cast Engineering, 2021. https://mc-castengineering.com/reports-blog-en/2019/11/27/why-is-there-water-in-my-air-compressor.

[12]"All about Oil lubricated Air Compressor Technology | CompAir", Compair.com, 2021. https://www.compair.com/en-pk/technologies/oil-lubricated-air-compressor.

[13] GHH RAND, Air and Gas Compression Solutions and Services. Ingersoll Rand Industrial Technologies. https:/www.kompresory-servis.sk/files/ghh-rand/ghh-product-brochure-en.pdf.

[14] VMI International AB, VIBER $\quad$ X5 MKIII TM $^{\mathrm{TM}}$ Instrument 2015. https://www.vmiab.com/res/portable/viberx5/manual_viberx5mkIII.pdf. 